\title{
Natural Pupil Size and Ocular Aberration under Binocular and Monocular Conditions
}

\author{
Takushi Kawamorita ${ }^{1,2 *}$ and Hiroshi Uozato ${ }^{1,2}$
}

${ }^{1}$ Department of Orthoptics and Visual Science, Kitasato University School of Allied Health Sciences, Japan

${ }^{2}$ Department of Ophthalmology and Visual Science, Kitasato University Graduate School of Medical Sciences, Japan

\begin{abstract}
Purpose: To investigate how activity of natural pupils under binocular and monocular conditions affect wave front aberrations.

Materials and Methods: Eighteen eyes from 18 subjects (mean age $22.3 \pm 0.8$ years) were included in the study. The undilated pupil diameters under photopic conditions were measured using the FP-10000 (TMI, Japan) infrared pupillometer. Aberrometry measurements were performed using the KR-9000PW (Topcon, Japan) HartmannShack wavefront sensor. Zernike coefficients were recalculated for the diameters of each pupil under binocular and monocular conditions using Schwiegerling's algorithm. Multiple regression analysis was performed to analyze independent predictors of the change of higher-order aberration for $6.0 \mathrm{~mm}$ from the binocular to the monocular condition. The independent variables were the change of pupil diameter from binocular to monocular condition; binocular pupil diameter; total higher-order aberration for $6.0 \mathrm{~mm}$, sphere, and cylinder

Results: Pupil diameter, total, total higher-order, coma-like, and spherical-like aberrations under monocular conditions were significantly greater than the binocular condition (all $P<0.01$ ). The multiple regression of analysis of variables showed that the change of total higher order aberration from the binocular to the monocular condition was related to the change of pupil diameter, and the amount of higher-order aberrations for $6.0 \mathrm{~mm}(P<0.05)$.

Conclusions: The outcomes suggest that increased pupil diameter under monocular conditions produces higher wavefront aberrations than under binocular conditions, resulting in a degradation of retinal image quality. This effect is enhanced in eyes with greater higher order aberrations and pupil diameter.
\end{abstract}

Keywords: Pupil size; Wavefront aberrations; Binocular condition; Monocular condition; Schwiegerling's algorithm

\section{Introduction}

Increasingly the effect of pupil diameter is being incorporated into cataract surgery, refractive surgery [1-4] and the contact lens fittings [5]. This has led to the introduction of infrared pupillometers and the incorporation pupillometry functions into wave front analyzers and corneal topographers $[6,7]$.

Clinically, visual performance is simulated for monocular conditions however, pupil diameter under monocular conditions is greater than under "real life" binocular conditions [8]. This pupilenlargement effect leads to an increase in the optical aberrations, therefore, the evaluation of visual performance under binocular conditions is important. A number of studies have advocated the importance evaluating post-refractive surgery patients under binocular conditions [9-12]. However, it is unclear how this change in physiologic pupil diameter between binocular and the monocular conditions affects the increase in ocular wavefront aberrations or whether the change depends on pupil size, the magnitude of higher-order aberration, or refractive error.

The current study investigated how the activity of natural pupils under the binocular and monocular conditions affects higher-order wavefront aberrations and whether there is dependency on aberration levels and/or refractive error.

\section{Materials and Methods}

Eighteen eyes from 18 subjects (aged 20-24 years; mean $22.3 \pm$ 0.8 years) with no known ocular abnormalities were included in this study. Mean sphere was $-5.40 \pm 3.26 \mathrm{D}$ (range: 0 to $-13.25 \mathrm{D}$ ), mean cylinder was $-0.50 \pm 0.58 \mathrm{D}$ (range: 0.00 to $-2.00 \mathrm{D}$ ), mean spherical equivalent refraction was $-5.65 \pm 3.36 \mathrm{D}$ (range: 0.00 to $-13.38 \mathrm{D}$ ). The exclusion criteria included distance Best Corrected Visual Acuity (BCVA) (LogMAR) of worse than 0 in either eye, and wearing hard contact lenses or spectacles. Pupil measurements were not performed with spectacles on because they significantly affect pupil magnification or distort infrared light (IR) used in most pupillometers. The tenets of the Declaration of Helsinki were followed and informed consent was obtained from all subjects in the current study.

\section{Pupil measurements}

Physiologically dilated horizontal pupil diameters were measured using the FP- $10000^{\circ}$ (TMI, Saitama, Japan) infrared electronic pupillometer that was connected to a laptop computer with proprietary pupil analysis software (TMI, version 1.08). The sampling rate was 30 Hz. Emmetropic eyes were measured without correction, and myopic eyes were measured with soft contact lenses on. All measurements were performed under photopic conditions with an ambient illuminance

*Corresponding author: Takushi Kawamorita, CO, Department of Orthoptics and Visual Science, Kitasato University School of Allied Health Sciences, 1-151 Kitasato, Minami, Sagamihara, Kanagawa, 252-0373, Japan, Tel: +81-42-7789669; Fax: +81-42-9669; E-mail: kawa2008@kitasato-u.ac.jp

Received December 13, 2013; Accepted December 29, 2013; Published January 06, 2014

Citation: Kawamorita T, Uozato H (2014) Natural Pupil Size and Ocular Aberration under Binocular and Monocular Conditions. J Comput Sci Syst Biol 7: 015-019. doi:10.4172/jcsb.1000133

Copyright: ( 2014 Kawamorita T, et al. This is an open-access article distributed under the terms of the Creative Commons Attribution License, which permits unrestricted use, distribution, and reproduction in any medium, provided the original author and source are credited. 
Citation: Kawamorita T, Uozato H (2014) Natural Pupil Size and Ocular Aberration under Binocular and Monocular Conditions. J Comput Sci Syst Biol 7: 015-019. doi:10.4172/jcsb.1000133

of $520 \mathrm{~lx}$ measured using an illuminance meter (T-10, Minolta Corp, Tokyo, Japan). The FP- $10000^{\circ}$ can measure pupil diameter in real time under binocular conditions which closely simulate natural viewing conditions (Figure 1). The magnitude of error introduced by variation in the vertex distance between the cornea and FP-10000 using the circular apertures of $3.0 \mathrm{~mm}$ and $5.6 \mathrm{~mm}$ was calculated. The repeatability of the two measurements by the same examiner was determined in 10 eyes. Evaluation of repeatability were assessed using the method described by Bland and Altman: the mean difference and the $95 \%$ limits of agreement (LoA) [13].

\section{Ocular wavefront aberration measurements and computation to natural pupil sizes}

Aberrometry measurements were performed with the KR9000 $\mathrm{PW}^{\circ}$ (Topcon, Tokyo, Japan) Hartmann-Shack wavefront sensor. Wavefront aberrations were analyzed at a $6.0 \mathrm{~mm}$ pupil diameter for total $(\mathrm{S} 2 \sim \mathrm{S} 6)$, total higher-order (S3 S6), coma-like (S3+S5), and spherical-like (S4+S6) aberrations. The magnitude of the Zernike polynomial coefficients were represented as the root mean square (RMS) [14]. Zernike coefficients were recalculated for each pupil diameter for binocular and the monocular conditions, using Schwiegerling's algorithm for recalculating expansion coefficients for an arbitrary pupil size for a $6.0 \mathrm{~mm}$ pupil diameter [15].

\section{Procedure}

Ten minutes were allowed for adaptation to the room illuminance prior to the measurements. A crisscross fixation target of 1 degree in the central visual field was placed at a distance of $5.0 \mathrm{~m}$. Under binocular conditions pupil diameters of the right eye were continuously measured for 10 seconds and averaged. Subsequently the left eye was occluded with a black patch, and after two minutes, the pupil diameter of the right eye was again continuously measured for 10 seconds and averaged. The effects of blinking were disregarded.

\section{Data Analysis}

Wilcoxon's signed rank sum test and multiple regression analyses using StatView version 5.0 software (SAS, Cary, USA) were performed. Multiple regression was performed to analyze the impact of binocular to monocular conditions on the change in higher-order aberrations. The independent variables were the change in pupil diameter from binocular to monocular conditions, binocular pupil diameter, total higher-order aberrations for a $6.0 \mathrm{~mm}$ pupil, and refractive error (sphere and cylinder). A $P<0.05$ was considered statistically significant.

\section{Results}

The relative error introduced by variation in the vertex distance between the cornea and the FP- 10000 pupillometer was 0 to $5 \%$ (Figure 2). Under photopic conditions, the mean difference between repeated measurements was $0.07 \mathrm{~mm}$ and the $95 \%$ LoA were -0.51 to $+0.65 \mathrm{~mm}$ for binocular measurements, and the mean difference was $-0.16 \mathrm{~mm}$ and -0.70 to $0.39 \mathrm{~mm}$ ( $95 \% \mathrm{LoA})$ for monocular measurements.

Mean RMS was $0.46 \pm 0.19 \mu \mathrm{m}$ for total higher order aberrations, $0.36 \pm 0.13 \mu \mathrm{m}$ for coma-like aberrations and $0.25 \pm 0.17 \mu \mathrm{m}$ for spherical-like aberrations. Mean pupil diameter under significantly increased under monocular conditions compared to the binocular conditions $(P<0.001$, Table 1$)$. Additionally total wavefront aberrations, total higher-order, coma-like, spherical-like aberrations all increased significantly (all $P<0.001$, Table 1$)$.
Multiple regression analysis of variables $(P=0.0005$, adjusted

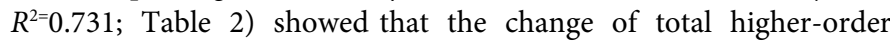
aberrations from the binocular to the monocular condition: Equation 1: monocular RMS - binocular RMS was related to the change of pupil diameter $(P<0.0001)$, and the magnitude of total higher-order aberrations $(P=0.038)$ for a $6.0 \mathrm{~mm}$ pupil diameter (Table 2$)$. There was no correlation to the diameter the binocular pupil $(P=0.356)$, sphere $(P=0.572)$ or cylinder, $(P=0.367)$ (Table 2$)$.

Two eyes were selected as sample eyes to demonstrate the data acquired for 330 seconds with the pupillometer. One eye had relatively

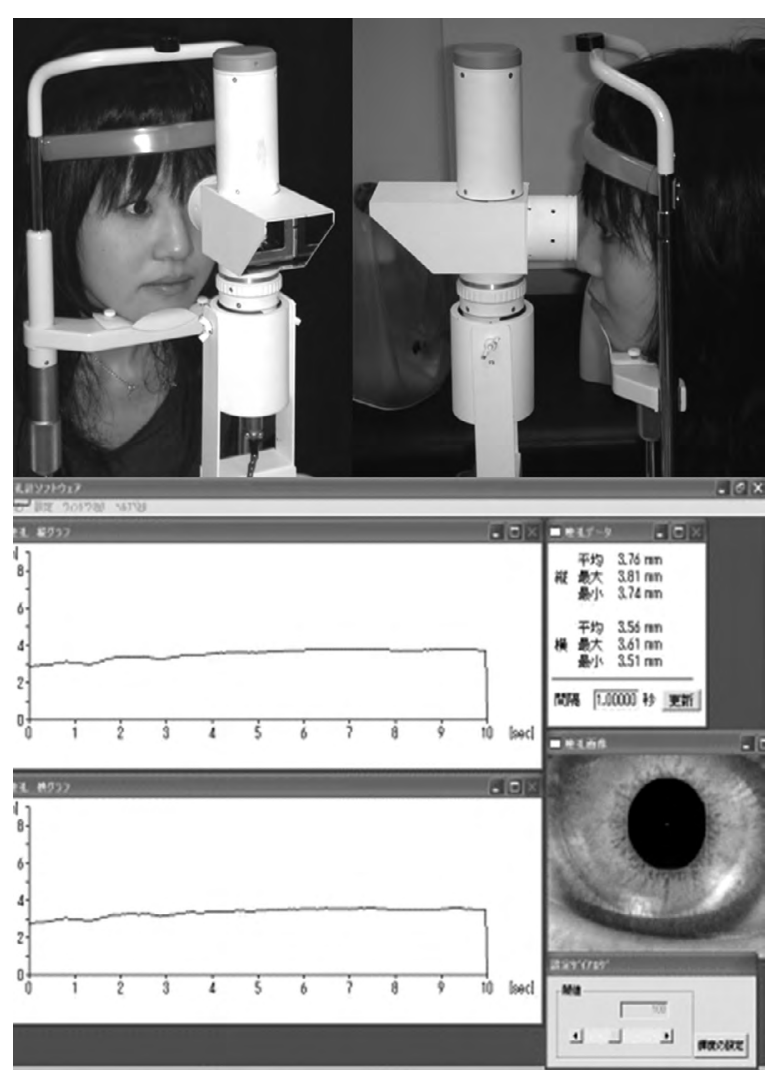

Figure 1: Photograph of the FP-10000.

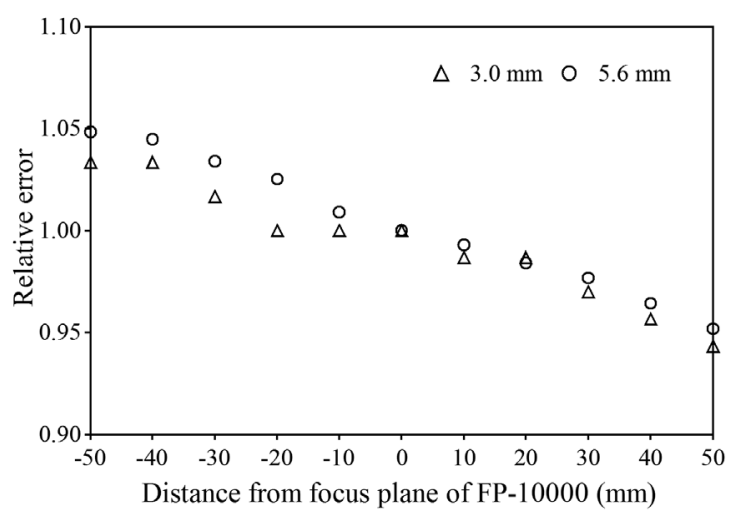

Figure 2: The relative error introduced by variation in the vertex distance between the corneal and the pupillometer's sensing equipment with the circular aperture $3.0 \mathrm{~mm}$ and $5.6 \mathrm{~mm}$. 


\begin{tabular}{|l|c|c|}
\hline Measurement & Binocular Condition & Monocular Condition \\
\hline PD $(\mathrm{mm})$ & $3.973 \pm 0.698$ & $5.059 \pm 0.933$ \\
\hline Computed wavefront aberration (um) & & \\
\hline Total aberration & $2.962 \pm 3.916$ & $3.294 \pm 6.476$ \\
\hline Total higher order aberration & $0.164 \pm 0.114$ & $0.322 \pm 0.247$ \\
\hline Coma-like abarration & $0.125 \pm 0.051$ & 0.001 \\
\hline Spherical-like aberration & $0.092 \pm 0.115$ & $<.001$ \\
\hline
\end{tabular}

Data are expressed as mean \pm standard deviation

$\mathrm{PD}=$ pupil diameter

Table 1: Mean Pupil Diameters and Wave front Aberrations under the Binocular and the Monocular Conditions

\begin{tabular}{|l|c|c|c|}
\hline Explanatory variables & Regression coefficients (SE) & Standardized coefficients & P Value \\
\hline Intercept & $-0.368(0.148)$ & -0.368 & 0.029 \\
\hline Mocular PD - Binocular PD (mm) & $0.242(0.042)$ & 0.764 & -0.692 to -0.045 \\
\hline Binocular PD (mm) & $0.033(0.034)$ & 0.151 to 0.333 & 0.129 \\
\hline Total higher order aberration for 6.0mm (um) & $0.315(0.135)$ & 0.311 & 0.356 \\
\hline Sphere (D) & $0.005(0.008)$ & 0.042 to 0.128 & 0.038 \\
\hline Cylinder (D) & $-0.040(0.043)$ & -0.131 & 0.572 \\
\hline
\end{tabular}

$\mathrm{P}=0.0005$, Adjusted $\mathrm{R}^{2}=0.731$

$\mathrm{SE}=$ Standard Error; $\mathrm{Cl}=$ Confidence Interval; $\mathrm{PD}=$ Pupil Diameter

Dependent variable is the change of the total higher order aberration from the binocular to the monocular condition

Table 2: Results of Multiple Regressions.

low values and/or changes in ocular aberration (Subject 1) (defocus value $3.60 \mu \mathrm{m}$ ) and pupil diameter, and the other eye, had higher values (Subject 2) (defocus value $14.73 \mu \mathrm{m}$ ) (Figures $3 \mathrm{~A}$ and $3 \mathrm{~B}$, respectively). Once the fellow eye was occluded, the pupil diameter in the right eye increased within 30 seconds and remained maximally dilated for 3 minutes or longer (Figure $3 \mathrm{~A}$ and $3 \mathrm{~B}$ ). The pupil diameter in subject 2 gradually decreased after approximately 3 minutes (Figure 3B). In subject 2 there were concomitant changes in higher order aberrations that follow the changes in pupil diameter (Figure 3B). For Subject 2, the increases and decreases in lower-order aberrations followed the changes in pupil diameter (Figure 4).

\section{Discussion}

The outcomes from the current study revealed that; (1) as has been reported previously [8], pupil and ocular wavefront aberrations are larger under monocular viewing conditions than binocular viewing conditions; (2) eyes with larger wavefront aberrations and/or pupil diameters are more significantly affected by this change; and (3) the change of the total higher-order aberrations from the binocular to the monocular condition does not depend on refractive error. Secondarily we confirmed that under photopic conditions, the FP-10000 had good repeatability and introduced very low error due to variation in the vertex distance between the cornea and the pupillometer.

An increase in the pupil diameter will generally cause an increase in optical aberrations [14] causing distortions in the point- and line spread functions [16]. A reduction in modulation transfer function (MTF) and decrease in the Strehl ratio all of which indicate diminished retinal image quality $[17,18]$. Clinically, the change in the pupil diameter would affect measures of subjective visual performance such as visual acuity, contrast sensitivity and on the like. Our results suggest that the larger the wavefront aberrations, the greater the effects would be. Therefore, eyes that have undergone corneal surgery [19], cataract surgery [20], or eyes with corneal pathology (eg. dry eye) [21] and keratoconus [22] that have higher than normal optical aberrations and are depend on pupil diameters, would be affected by pupil enlargement.

Reduction in visual performance after photorefractive keratectomy and laser in situ keratomileusis (LASIK) have been previously reported $[23,24]$. However, the visual performance under monocular conditions in the laboratory would be an underestimate, a worse evaluation, as compared with that in real life. Additionally, this pupil-enlargement phenomenon might have a significant effect on uncorrected visual performance, especially in myopic eyes as shown in Figure 4 and previous studies [25]. Cuesta et al. [10] and Anera et al [9] reported that interocular differences in ocular aberrations and corneal asphericity affect binocular visual function and should be considered in refractive surgery. Therefore the pupil-enlargement phenomenon described in the current study also affects interocular difference, having important implications for refractive surgery.

As the wavefront aberrations increase under monocular viewing conditions, the larger pupil diameter would theoretically increase the retinal illuminance. This indicates that other factors may mitigate the effects of increased aberrations. Analyzing post-LASIK (with presumably higher wavefront aberrations) and non-LASIK groups, Boxer Wachler [8], found no differences in the improvement in contrast sensitivity and visual acuity from the binocular to monocular testing conditions. However, the optical aberrations were not reported in Boxer-Wachler's study and measurements of visual performance were tested under dim-lighting conditions unlike our study. Oshika et al. [26] reported that increased spherical aberration in pre and post-LASIK eyes with larger photopic pupils, predominately affected contrast sensitivity. These conflicting results indicate that further studies are required to determine the effect of pupil enlargement on subjective visual performance, such as low contrast visual acuity and mesopic contrast sensitivity. Further studies are also required to clarify the effect of hippus and accommodative miosis [27], and whether the pupil size changes with time as shown in Subject 2 (Figures $3 \mathrm{~B}$ and 4), which may lead to inconsistent results during assessment of visual performance.

A limitation of the current study was that the pupillometer will slightly reduce the illuminance of the eye being tested. The values we obtained under photopic conditions were larger than those reported previously [28]. Likely, the pupil enlargement effect might have to 


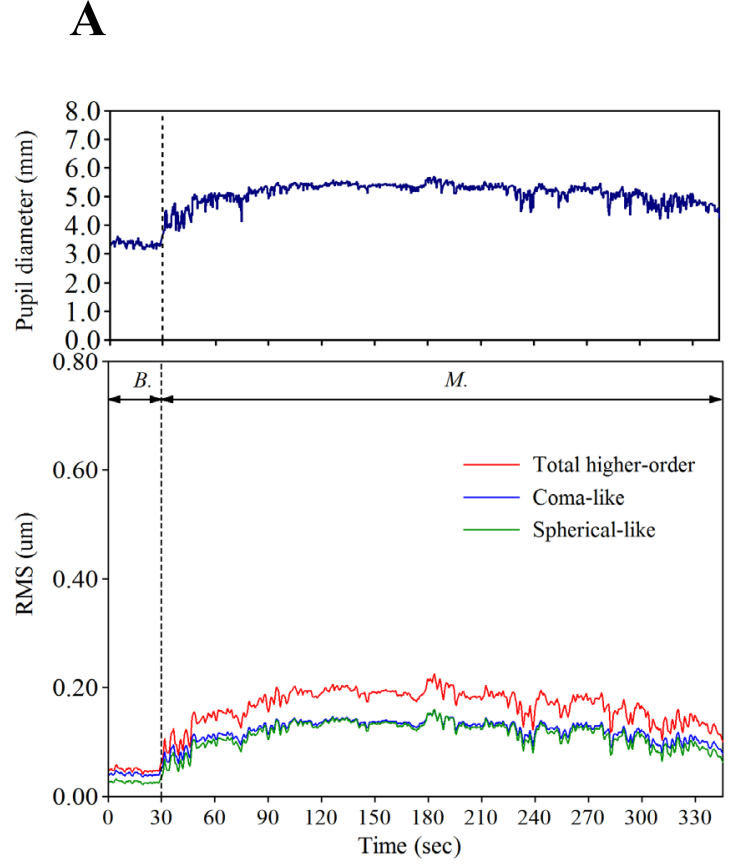

B

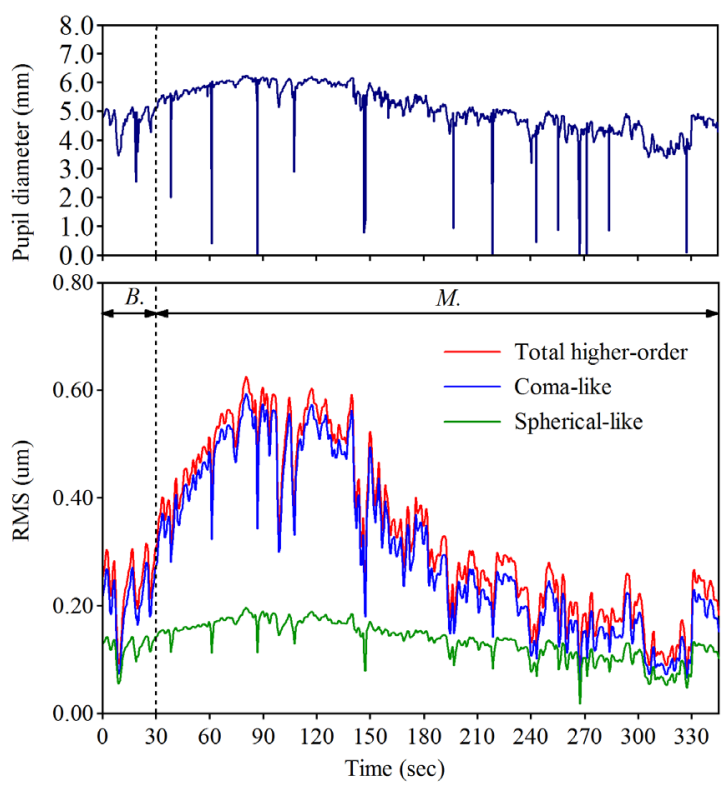

Figure 3: Time course of the pupil diameters (Top) and the higher-order aberrations (total higher-order, coma-like, spherical-like aberration; Below) computed with Schwiegerling's algorithm based on the natural pupil diameters and wavefront aberrations for $6.0 \mathrm{~mm}$ from the binocular to the monocular condition in A) subject 1 , who had particularly small values and/ or changes in wavefront aberrations and pupil diameters and B) subject 2 , whose values/changes were large. The vertical dash shows the time the non-measurement eye was covered. (B.=binocular condition, M.=monocular condition, RMS=root mean square).

be considered as a function of the state of retinal adaptation in the occluded eye even under photopic conditions as retinal adaptation affects pupil size [29,30]. We did not incorporate the effects of retinal adaptation in the current study. We did not account for the change in

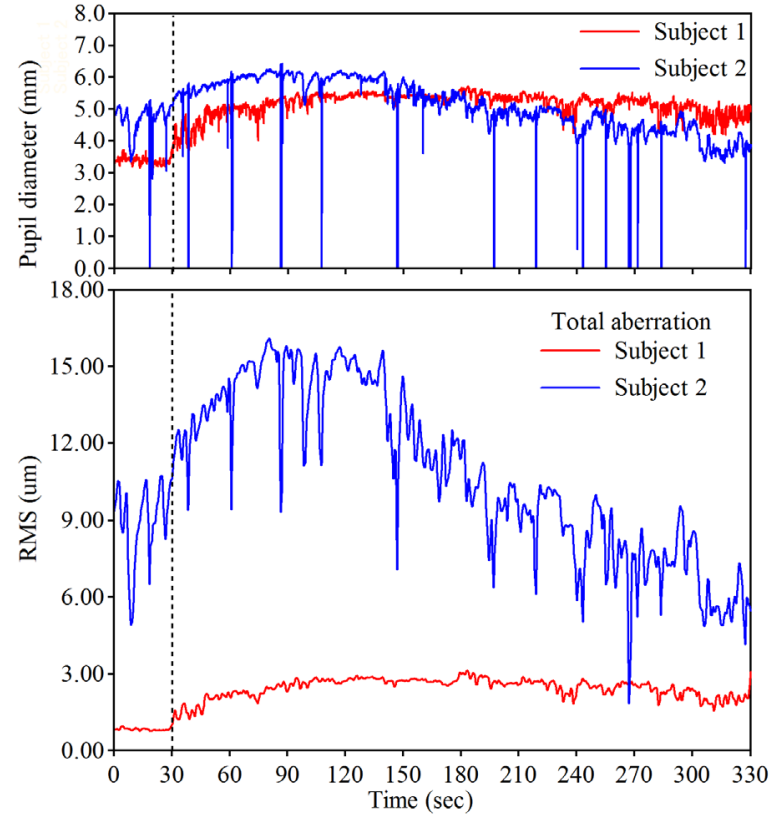

Figure 4: Time course of the total aberrations (including low-order aberrations) computed with Schwiegerling's algorithm based on the natural pupil diameters and wavefront aberrations for $6.0 \mathrm{~mm}$ from the binocular to the monocular condition in subject 1 and subject 2 . The vertical dash shows the time the non-measurement eye was covered. (B.=binocular condition, $\mathrm{M} .=$ monocular condition, $\mathrm{RMS}=$ root mean square).

pupil center due changes in pupil size and the effect on the magnitude or pattern of higher order aberrations. Walsh reported that even for a 3 $\mathrm{mm}$ pupil diameter, shifts in the pupil center of $0.2 \mathrm{~mm}$ can change the MTF up to $50 \%$ at high spatial frequencies. The change in MTF would be greater, eyes with larger shifts in pupil centration from binocular to monocular conditions [31]. Further limitations of the current study include investigation of monochromatic aberrations, and small sample size, especially the lack of hyperopic eyes.

In conclusion, increased pupil diameter under monocular conditions increases higher optical aberrations, resulting in worse retinal image quality. In eyes with higher optical aberrations and pupil diameter this effect is likely enhanced. The outcomes from the current study show the importance of measuring pupil diameter under both binocular and monocular conditions, especially in eyes with larger optical aberrations. Practically, pupil diameter should be measured under binocular conditions.

\section{Acknowledgments}

The authors thank Dr. Joseph M. Miller and Dr. Jim Schwiegerling for teaching and encouragement. This study was supported by a grant from Kitasato University Research Grant for Young Researchers 2011, 2012, and 2013) (T.K.)

\section{References}

1. Hiatt JA, Grant CN, Boxer Wachler BS (2005) Establishing analysis parameters for spherical aberration after wavefront LASIK. Ophthalmology 112: 998-1002.

2. Kawamorita $\mathrm{T}$, Uozato $\mathrm{H}(2005)$ Modulation transfer function and pupil size in multifocal and monofocal intraocular lenses in vitro. J Cataract Refract Surg 31: 2379-2385.

3. Koch DD, Samuelson SW, Haft EA, Merin LM (1991) Pupillary size and responsiveness. Implications for selection of a bifocal intraocular lens. Ophthalmology 98: 1030-1035.

4. Koch DD, Samuelson SW, Villarreal R, Haft EA, Kohnen T (1996) Changes 
Citation: Kawamorita T, Uozato H (2014) Natural Pupil Size and Ocular Aberration under Binocular and Monocular Conditions. J Comput Sci Syst Biol 7: 015-019. doi:10.4172/jcsb.1000133

in pupil size induced by phacoemulsification and posterior chamber lens implantation: consequences for multifocal lenses. J Cataract Refract Surg 22 579-584.

5. Pujol J, Gispets J, Arjona M (2003) Optical performance in eyes wearing two multifocal contact lens designs. Ophthalmic Physiol Opt 23: 347-360.

6. Kohnen T, Terzi E, Kasper T, Kohnen EM, Bühren J (2004) Correlation of infrared pupillometers and CCD-camera imaging from aberrometry and videokeratography for determining scotopic pupil size. J Cataract Refract Surg 30: 2116-2123.

7. Spadea L, Giammaria D, Ferrante R, Balestrazzi E (2005) Pre-excimer laser and post-excimer laser refractive surgery measurements of scotopic pupil diameter using 2 pupillometers. Ophthalmology 112: 1003-1008.

8. Boxer Wachler BS (2003) Effect of pupil size on visual function under monocula and binocular conditions in LASIK and non-LASIK patients. J Cataract Refract Surg 29: 275-278.

9. Anera RG, Jiménez JR, Villa C, Rodríguez-Marín F, Gutiérrez R (2007) Technical note: Pre-surgical anisometropia influences post-LASIK binocular mesopic contrast sensitivity function. Ophthalmic Physiol Opt 27: 210-212.

10. Cuesta JR, Anera RG, Jiménez R, Salas C (2003) Impact of interocular differences in corneal asphericity on binocular summation. Am J Ophthalmol 135: $279-284$.

11. Jiménez JR, Villa C, Anera RG, Gutiérrez R, del Barco LJ (2006) Binocular visual performance after LASIK. J Refract Surg 22: 679-688.

12. Lombardo M, Lombardo G, Serrao S (2006) Interocular high-order corneal wavefront aberration symmetry. J Opt Soc Am A Opt Image Sci Vis 23: 777787

13. Bland JM, Altman DG (1986) Statistical methods for assessing agreement between two methods of clinical measurement. Lancet 1: 307-310.

14. Howland HC (2002) High order wave aberration of eyes. Ophthalmic Physio Opt 22: 434-439.

15. Schwiegerling J (2002) Scaling Zernike expansion coefficients to different pupil sizes. J Opt Soc Am A Opt Image Sci Vis 19: 1937-1945.

16. Campbell FW, Green DG (1965) Optical and retinal factors affecting visual resolution. J Physiol 181: 576-593.

17. Guirao A, González C, Redondo M, Geraghty E, Norrby S, et al. (1999) Average optical performance of the human eye as a function of age in a normal population. Invest Ophthalmol Vis Sci 40: 203-213.
18. Laughlin SB (1992) Retinal information capacity and the function of the pupil. Ophthalmic Physiol Opt 12: 161-164.

19. Aizawa D, Shimizu K, Komatsu M, Ito M, Suzuki M, et al. (2003) Clinical outcomes of wavefront-guided laser in situ keratomileusis: 6-month follow-up. J Cataract Refract Surg 29: 1507-1513.

20. Miller JM, Anwaruddin R, Straub J, Schwiegerling J (2002) Higher order aberrations in normal, dilated, intraocular lens, and laser in situ keratomileusis corneas. J Refract Surg 18: S579-583.

21. Montés-Micó R, Alió JL, Charman WN (2005) Dynamic changes in the tear film in dry eyes. Invest Ophthalmol Vis Sci 46: 1615-1619.

22. Applegate RA, Hilmantel G, Howland HC, Tu EY, Starck T, et al. (2000) Corneal first surface optical aberrations and visual performance. J Refract Surg 16: 507-514.

23. Seiler T, Kaemmerer M, Mierdel P, Krinke HE (2000) Ocular optical aberrations after photorefractive keratectomy for myopia and myopic astigmatism. Arch Ophthalmol 118: 17-21.

24. Yamane N, Miyata K, Samejima T, Hiraoka T, Kiuchi T, et al. (2004) Ocular higher-order aberrations and contrast sensitivity after conventional laser in situ keratomileusis. Invest Ophthalmol Vis Sci 45: 3986-3990

25. Atchison DA, Smith G (2002) Optics of the human eye. Edinburgh, UK Butterworth-Heinemann

26. Oshika T, Tokunaga T, Samejima T, Miyata K, Kawana K, et al. (2006) Influence of pupil diameter on the relation between ocular higher-order aberration and contrast sensitivity after laser in situ keratomileusis. Invest Ophthalmol Vis Sc 47: $1334-1338$.

27. Brown S, Bradley J (2010) Decreased pupil diameter with digital infrared pupillometer. J Cataract Refract Surg 36: 874-875.

28. Winn B, Whitaker D, Elliott DB, Phillips NJ (1994) Factors affecting lightadapted pupil size in normal human subjects. Invest Ophthalmol Vis Sci 35 1132-1137.

29. Alpern M, Ohba N (1972) The effect of bleaching and backgrounds on pupi size. Vision Res 12: 943-951.

30. Brown SM, Khanani AM, Xu KT (2004) Day to day variability of the darkadapted pupil diameter. J Cataract Refract Surg 30: 639-644.

31. Walsh G (1988) The effect of mydriasis on the pupillary centration of the human eye. Ophthalmic Physiol Opt 8: 178-182. 